\title{
Antifungal resistance: current trends and future strategies to combat
}

This article was published in the following Dove Press journal:

Infection and Drug Resistance

29 August 2017

Number of times this article has been viewed

\section{Nathan P Wiederhold \\ Department of Pathology and Laboratory Medicine, Fungus Testing Laboratory, University of Texas Health Science Center at San Antonio, San Antonio, TX, USA}

\begin{abstract}
Antifungal resistance represents a major clinical challenge to clinicians responsible for treating invasive fungal infections due to the limited arsenal of systemically available antifungal agents. In addition current drugs may be limited by drug-drug interactions and serious adverse effects/toxicities that prevent their prolonged use or dosage escalation. Fluconazole resistance is of particular concern in non-Candida albicans species due to the increased incidence of infections caused by these species in different geographic locations worldwide and the elevated prevalence of resistance to this commonly used azole in many institutions. C. glabrata resistance to the echinocandins has also been documented to be rising in several US institutions, and a higher percentage of these isolates may also be azole resistant. Azole resistance in Aspergillus fumigatus due to clinical and environmental exposure to this class of agents has also been found worldwide, and these isolates can cause invasive infections with high mortality rates. In addition, several species of Aspergillus, and other molds, including Scedosporium and Fusarium species, have reduced susceptibility or pan-resistance to clinically available antifungals. Various investigational antifungals are currently in preclinical or clinical development, including several of them that have the potential to overcome resistance observed against the azoles and the echinocandins. These include agents that also target ergosterol and $\beta$-glucan biosynthesis, as well as compounds with novel mechanisms of action that may also overcome the limitations of currently available antifungal classes, including both resistance and adverse effects/toxicity. Keywords: azoles, echinocandins, Aspergillus, Candida albicans, investigational antifungals, non-albicans Candida species, acquired resistance, intrinsic resistance, Candida auris
\end{abstract}

\section{Introduction}

Antifungal resistance is becoming a significant concern to clinicians who are charged with caring for patients at high risk for invasive mycoses. Resistance to currently available antifungal agents can develop secondary to acquired mechanisms following exposure to these drugs. Recent trends in acquired antifungal resistance include increased azole resistance among non-Candida albicans isolates, azoles resistance in Aspergillus fumigatus, and echinocandin resistance in C. glabrata $^{1-3}$ In contrast, some fungal species are intrinsically resistant to certain drugs (e.g., C. krusei and fluconazole, or $C$. lusitaniae and amphotericin B), while others demonstrate microbiologic resistance to all clinically available antifungals (e.g., Lomentospora [formerly Scedosporium] prolificans and Fusarium solani). ${ }^{4-6}$ New species are also emerging that may demonstrate resistance to multiple class of available agents (e.g., C. auris). ${ }^{7}$ Although the prevalence of antifungal resistance is not at the levels observed for some bacteria against different 
antibiotics, treatment options for invasive fungal infections are limited, and patients at highest risk often have multiple comorbidities, including immunosuppression, which may limit the effectiveness of therapy even in the absence of drug resistance. Clearly, new treatment strategies are needed to address this issue, in addition to overcoming the toxicities/ adverse effects and drug interactions that are associated with currently available antifungals, which themselves can limit the effectiveness of therapy. Several new antifungals are currently under preclinical and clinical evaluation that may help to address the problem of antifungal resistance. The purpose of this review is to discuss the current trends in antifungal resistance and new antifungals currently under preclinical development and in clinical trials that may improve outcomes in patients with invasive fungal infections. In addition, numerous extracts from different plants have also been shown to have activity against various fungi, including isolates resistant to currently available antifungal agents. However, a detailed discussion of medicinal plants and their extracts is beyond the scope of this review.

\section{Resistance in non-C. albicans}

\section{Azole resistance}

Although C. albicans is the most common Candida species cultured from patients with candidiaisis, infections caused by other species within this genus are becoming more important in various regions around the world, including C. glabrata, C. parapsilosis, and C. tropicalis, and the species can vary between different geographic regions. This is of importance, as resistance has been shown to be increasing in many of the non-C. albicans species in different institutions and geographic regions. ${ }^{8-10}$ As reported by the World Health Organization, fluconazole resistance is indeed more common in non-C. albicans species (Figure 1). ${ }^{11}$ This is of concern, since fluconazole is a relatively inexpensive and well-tolerated medication that is easily administered orally. Furthermore, resistance to fluconazole may also mean resistance to other azoles, since mechanisms that reduce fluconazole susceptibility, such as point mutations within the ERG11 gene that encodes lanosterol $14 \alpha$-demethylase, the target of the azoles (e.g., itraconazole, voriconazole, posaconazole, and isavuconazole), increase transcription of this gene, leading to increased amounts of the enzyme, or the efflux pumps, such as Cdr1 and Cdr2, also affect this class of antifungals. ${ }^{12,13}$

As previously noted, the predominant non-C. albicans species causing infections may vary between different geographic regions, and the rates of azole resistance may also differ between institutions. This may be influenced by the prescribing patterns of clinicians for both the treatment of and prophylaxis against invasive candidiasis. ${ }^{14}$ In the USA, C. glabrata is the second most common cause of invasive candidiasis, and fluconazole resistance has been reported as high as $12 \%-18 \%$ in some institutions. ${ }^{15,16}$ In contrast, in some health care institutions in India, C. tropicalis is the predominant species, and rates of fluconazole resistance may vary significantly. ${ }^{17,18}$ The prevalence of $C$. parapsilosis approaches that of $C$. albicans in some Chinese hospitals in terms of the number of isolates cultured from patients with invasive infections. ${ }^{19-22}$ Fluconazole susceptibility is also highly variable between institutions, with some reporting no azole resistance while others have reported that fluconazolesusceptible, dose-dependent plus resistant isolates may be as high as $50 \%$ in intensive care units. ${ }^{20,22}$

\section{Echinocandin resistance}

The echinocandins are recommended as the first line of therapy against invasive candidiasis in immunocompromised patients and in those who have had prior azole exposure due to the fear of resistance. ${ }^{23}$ Because the mechanism of action is different from that of the azoles, the echinocandins,

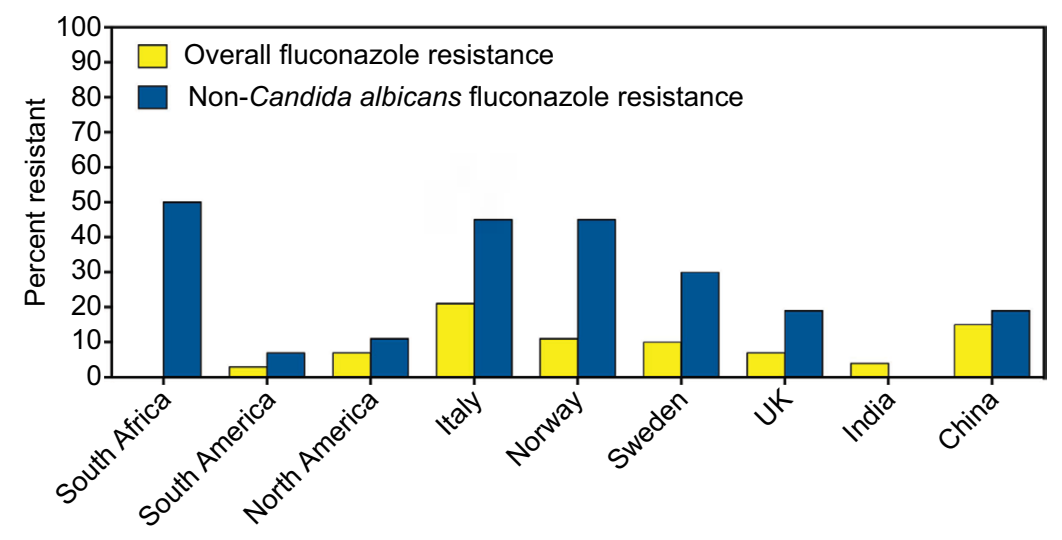

Figure I Fluconazole resistance overall and in non-Candida albicans isolates as per the World Health Organization. Note: Reprinted from World Health Organization. Antimicrobial Resistance: Global Report on Surveillance. Available from: http://www.who.int/drugresistance/documents/ surveillancereport/en/. Copyright 2014."1 
including anidulafungin, caspofungin, and micafungin, have been shown to maintain potent in vitro activity against many Candida isolates that have developed resistance to fluconazole and the other triazoles. ${ }^{24}$ However, resistance to the echinocandins can develop with exposure to the members of this class, and this occurs via point mutations within highly conserved regions (i.e., hot spots 1 and 2) of the FKS1 and $F K S 2$ genes, which encode subunits of the glucan synthase enzyme. ${ }^{25}$ These hot spot regions are conserved across different Candida species, and their detection has been reported in multiple species collected from patients who have experience both microbiologic and clinical failure. ${ }^{26-28}$

Although the overall rate of echinocandin resistance remains low, some institutions in the USA have reported increasing rates of infections caused by $C$. glabrata, which often occur in patients with multiple comorbidities. ${ }^{29}$ As with azole resistance, echinocandin resistance can develop with exposure to the members of this class. It has been suggested that echinocandin in vitro susceptibility results should be taken in context with a patient's history of exposure to these antifungals. In one study, the clinical failure rate approached $90 \%$ in patients who had prior echinocandin exposure and from whom a resistant $C$. glabrata isolate was isolated. ${ }^{30,31}$ In those whose infections were caused by echinocandin-susceptible isolates but with prior echinocandin exposure, clinical responses were still only $\sim 50 \%$. In addition to a patient's history of echinocandin exposure, the type of $F K S$ mutation may also play an important role in response to therapy and the likelihood of failing treatment with an echinocandin. In vitro studies have demonstrated that point mutations resulting in amino acid changes of serine to proline at codon 629 within Fks1p or codon 663 in Fks2p, as well as phenylalanine to serine at codon 659 in Fks2p in C. glabrata lead to reduced activity of the glucan synthase enzyme, ${ }^{32}$ which have translated into reduced in vivo efficacy of echinocandin therapy in an animal model of invasive candidiasis. ${ }^{33}$ Results from single-center, retrospective studies also suggest that this may have clinical consequences, as the majority of patients with infections caused by isolates harboring S663F or S663P amino acid changes failed therapy, while many of those with infections caused by other point mutations responded to treatment with an echinocandin. ${ }^{15,16}$ Although the clinical data are limited and more robust studies are needed, there is the possibility that the identification of the specific point mutations causing microbiologic resistance along with a patient's history of echinocandin exposure may be used to predict the likelihood of response to echinocandin therapy.

\section{Multidrug-resistant Candida isolates}

Resistance to multiple classes of drugs is also a concern in some non-C. albicans species. In one publication from the SENTRY study, $11 \%$ of fluconazole-resistant bloodstream infections were also resistant to an echinocandin. ${ }^{34}$ More recently, in a large surveillance study conducted in four large metropolitan areas in the USA, an increase in echinocandin nonsusceptible $C$. glabrata isolates (e.g., isolates classified as intermediate or resistant) was reported. ${ }^{1}$ While these results are consistent with those from single-center studies, in this multicenter surveillance study, which included over 1300 isolates, a third of the isolates that were nonsusceptible to an echinocandin were also resistant to fluconazole, compared to only $8.1 \%$ of the isolates that were echinocandin susceptible. Because of the differences in the mechanisms of action and known mechanisms of resistance, the exact cause of azole and echinocandin coresistance in some C. glabrata isolates is unknown. As many patients with invasive candidiasis due to C. glabrata have multiple comorbidities, previous exposure to these antifungal classes may also play a role. In addition, a hypermutable phenotype has been reported in isolates with a disrupted $\mathrm{MSH} 2$ mismatch repair gene, which may be more likely to produce multidrug-resistant mutants. ${ }^{35}$

Recently, attention has focused on the emerging pathogen $C$. auris. First described in 2009 for an isolate collected from the external ear canal of a patient, $C$. auris has quickly spread to multiple countries in several continents and has become a significant clinical problem. In a recent retrospective review of the clinical history of 54 patients, most had multiple risk factors for invasive disease and candidemia was observed in $61 \%$. $^{7}$ Strikingly, the mortality rate in this series of patients was $59 \%$. Unfortunately, antifungal therapy may be limited, as up to $90 \%$ of the isolates may be resistant to fluconazole, and 50\% have elevated voriconazole minimum inhibitory concentrations (MICs), which is secondary to point mutations in ERG11 and ERG3. ${ }^{7,36}$ Interestingly, posaconazole and isavuconazole appear to maintain some in vitro potency, although the clinical significance of this remains unknown. Currently, the echinocandins are recommended for the treatment of $C$. auris infections. However, elevated MICs secondary to FKS mutations have been found in some isolates. ${ }^{36-38}$ Unfortunately, this species is often misidentified by commercially available, automated systems that use biochemical means for species identification. ${ }^{36,37}$

\section{Resistance in Aspergillus species}

Recent attention has also begun to focus on azole-resistant Aspergillus species, with particular interest in resistant 
A. fumigatus isolates. As in Candida species, resistance to the mold active triazoles, itraconazole, posaconazole, voriconazole, and isavuconazole can develop with prolonged clinical exposure. This has been well documented in the literature and can occur in patients with chronic pulmonary aspergillosis, where azole therapy is often administered to patients for years. ${ }^{39,40}$ This acquired resistance in $A$. fumigatus is caused by point mutations in the CYP51A gene, which encodes the Cyp51 enzyme responsible for the conversion of lanosterol to ergosterol. Different mutations can differentially affect the azoles, with some causing resistance to voriconazole and isavuconazole, some causing resistance to posaconazole and itraconazole, and others causing pan-azole resistance. ${ }^{40-42}$ In addition, it is now known that environmental exposure to the azoles, which are used in a variety of means, including agriculture to protect plants and crops, can also lead to the development of azole resistance. In these isolates, tandem base pair repeats (abbreviated TR) have been found within the promoter region of this gene in azole-resistant $A$. fumigatus isolates collected from the environment, in addition to the point mutations within the CYP51A gene. These include the $\mathrm{TR}_{34} /$ $\mathrm{L} 98 \mathrm{H}$, which causes pan-azole resistance, and $\mathrm{TR}_{46} / \mathrm{Y} 121 \mathrm{~F} /$ T289A, which causes reduced posaconazole potency and high-level resistance to voriconazole and posaconazole. ${ }^{43,44}$ Both of these mutations have been documented in isolates collected from patients with invasive aspergillosis without a history of prior azole exposure ${ }^{39,40,45}$ and in the environment where azoles or similar demethylase inhibitors are used as fungicides. ${ }^{2,41,46}$ Isolates harboring these mutations have also been documented in numerous countries around the world. ${ }^{2,3}$ In addition to azole resistance in A. fumigatus, several cryptic or sibling species within Aspergillus section Fumigati (e.g., Aspergillus lentulus, Aspergillus felis, Aspergillus parafelis, Aspergillus pseudofelis, Aspergillus pseudoviridinutans, Aspergillus udagawae), as well as other Aspergillus species in different sections (e.g., Aspergillus calidoustus, Aspergillus flavus, Aspergillus sydowii, Aspergillus terreus, Aspergillus versicolor) may have reduced or variable susceptibility to the azoles as well as other antifungals. ${ }^{47-50}$ Although $A$. fumigatus is the most frequently isolated species at many institutions, studies have reported that the prevalence of cryptic species may be as high as $11 \%-14.5 \% .^{51,52}$ Thus, proper species identification in addition to antifungal susceptibility testing may help to guide therapy. However, cryptic species are difficult to differentiate based solely on phenotypic and morphologic characteristics. $^{53}$

The emergence of azole resistance in A. fumigatus is problematic due to the limited treatment options against infections caused by these fungi. Although both amphotericin $\mathrm{B}$ and the echinocandins can be used to treat patients with invasive aspergillosis, each has its limitations. Amphotericin B deoxycholate is associated with clinically significant nephrotoxicity that may limit its use, and although nephrotoxicity may be reduced with the lipid formulations of this polyene, it can still occur, especially with higher doses or prolonged administration. ${ }^{54}$ Although the echinocandins avoid the toxicities observed with amphotericin B formulations, these agents are not recommended as monotherapy for invasive aspergillosis. ${ }^{55}$ In addition, both amphotericin B formulations and the echinocandins must be administered intravenously, which can be problematic when prolonged therapy is required.

\section{Other molds with reduced susceptibility or intrinsic resistance to antifungals}

Several other molds have reduced susceptibility or are intrinsically resistant to clinically available antifungal agents. Those that are often reported in epidemiologic studies to cause disease in immunocompromised hosts include Scedosporium species, Lomentospora (formerly Scedosporium) prolificans, and Fusarium species. Scedosporiosis is an invasive infection that can occur in persistently neutropenic patients, those with lymphopenia, and in lung transplant recipients. ${ }^{56-60}$ The primary route of infection is via the lungs, but dissemination, including to the central nervous system, can occur. Several different Scedosporium species can cause this invasive mycosis, including Scedosporium apiospermum, Scedosporium boydii (formerly Pseudallescheria boydii), Scedosporium aurantiacum, Scedosporium dehoogii, Scedosporium minutispora, and Scedosporium desertorum. ${ }^{4,61}$ Variable antifungal activity has been reported against these species for different antifungal agents, including the azoles, amphotericin B, and the echinocandins, ${ }^{4,62,63}$ while L . prolificans is resistant to all clinically available agents. ${ }^{61,63}$ Although some case reports have documented successful treatment with combination therapy that includes the antiparasitic agent miltefosine ${ }^{64,65}$ this strategy has not been evaluated in clinical studies to confirm efficacy.

Fusariosis is a significant cause of morbidity and mortality in immunocompromised hosts. Infections in highly immunocompromised patients, including neutropenic patients with leukemia or those receiving high-dose corticosteroids for graft-versus-host disease, are usually invasive and may disseminate through the bloodstream. ${ }^{59,66,67}$ Although numerous Fusarium species are capable of causing disease in both plants and animals, the majority of infections in humans 
are caused by those within the $F$. solani and Fusarium oxysporum species complexes. ${ }^{68}$ The prognosis in patients with invasive fusariosis is poor, but may be improving due to the availability of newer antifungals and formulations, including voriconazole and lipid amphotericin B. ${ }^{67}$ However, several Fusarium species have reduced in vitro susceptibility to various antifungal classes, while others, including $F$. solani, may be pan-antifungal resistant.

\section{New antifungals for the treatment of resistant fungi}

Several investigational antifungals are currently under preclinical and clinical evaluation. These include agents that are similar to clinically available antifungal classes in terms of their mechanisms of action, but may offer distinct advantages to these drugs. Several of these agents have moved from preclinical development to clinical trials in healthy volunteers and patients. Some of these agents may soon be available for clinical use. In addition, several new compounds with novel mechanisms of action that may overcome both the limitations of resistance to and adverse effects of clinically available antifungals are also under development.

\section{VT-I I 29,VT-I I 6I, and VT-I598 - fungal specific inhibitors of Cyp5 I}

One of the main limitations of the azole class of antifungals is the clinically significant drug-drug interactions that occur with the members of this class. In addition to inhibition of the fungal lanosterol $14 \alpha$-demethylase (aka Cyp51), the azoles can also inhibit cytochrome P450 (CYP 450) enzymes that are responsible for the metabolism of various substances, including numerous other drugs. In addition, some of the azoles (e.g., fluconazole, voriconazole, itraconazole, and isavuconazole) are also substrates of the CYP 450 enzymes, and therefore, drugs that inhibit or induce the activity of these enzymes can also lead to clinically significant changes in azole concentrations. To overcome the problem of drug-drug interactions associated with the azoles, Viamet Pharmaceuticals, Inc. (Durham, NC, USA) has replaced the triazole metal-binding group with a tetrazole that binds less avidly to the active site of the Cyp51 enzyme and mammalian CYP 450 enzymes, while also modifying the portion of the compound that is recognized by amino acids of the substrate-binding site within this enzyme. These modifications have resulted in compounds with more specific inhibition of fungal Cyp 51 compared to mammalian CYP 450 enzymes, and thus the potential for drug-drug interactions. ${ }^{69-71}$ Preclinical results of three agents with these modifications have been reported in the literature (VT-1129, VT-1161, and VT-1598; Figure 2), and one agent is currently in clinical studies (VT-1129). Each of these agents has potent activity against various yeast isolates, including C. albicans and non-C. albicans species, and Cryptococcus species. ${ }^{72-75}$ The potent in vitro activity observed with VT-1161 was also maintained against some
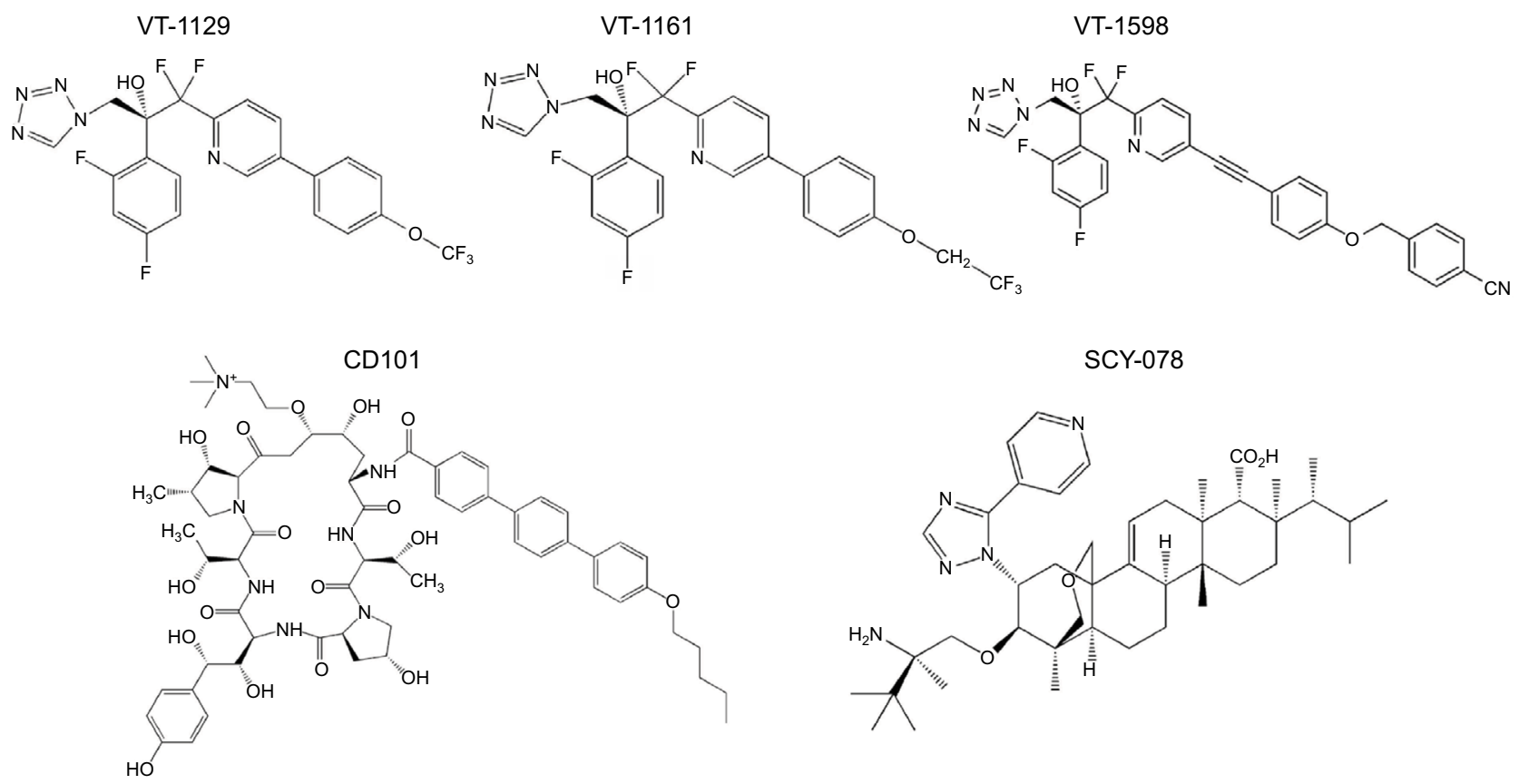

SCY-078

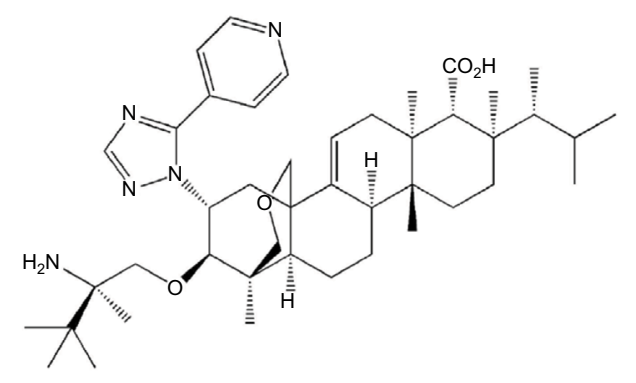

Figure 2 Investigational antifungal agents with mechanisms of action similar to that of the azoles via inhibition of ergosterol biosynthesis (VT-I I29, VT-II6I, VT-I598), or echinocandins via inhibition of I,3- $\beta$-D-glucan synthesis (CDIOI, SCY-078). 
fluconazole-resistant and echinocandin-resistant isolates, which has translated into in vivo efficacy in animal models of vulvovaginal and invasive candidiasis. ${ }^{72-78}$ Similar to VT-1161, VT-1129 also has potent in vitro activity against Candida and Cryptococcus species, and the activity of this investigational agent is also maintained against Cryptococcus gattii genotypes with reduced susceptibility to fluconazole. ${ }^{75}$ Of these three investigational agents, VT-1598 has the most potent activity against molds, including various Aspergillus species and Rhizopus arrhizus, as well as endemic fungi, including Coccidioides isolates, with elevated fluconazole MICs. ${ }^{79,80}$ This agent also demonstrates potent in vitro activity against other Candida species, including C. albicans, C. glabrata, C. parapsilosis, and C. tropicalis, although the potency was reduced against some $C$. albicans and $C$. glabrata isolates with elevated fluconazole MIC. ${ }^{81}$ Similarly, reduced in vitro potency has also been observed against some azole-resistant $A$. fumigatus isolates. However, the degree to which different mechanisms that cause azole resistance, including point mutations in ERG11/CYP51A or upregulation of efflux pumps, affect the in vitro activity or in vivo efficacy of VT-1598, VT-1161, or VT-1129 is not yet understood. This agent also maintains in vitro activity against some $C$. auris isolates. $^{82}$

\section{CDIOI and SCY-078 - inhibition of glucan synthase}

The echinocandins have the advantage of avoiding the drugdrug interactions associated with the azoles and the adverse effects/toxicities observed with amphotericin B formulations due to their inhibition of the fungal specific glucan synthase enzyme. However, as previously noted, C. glabrata resistance is increasingly being reported in some institutions. In addition, the echinocandins must be administered intravenously on a daily basis, thus prolonged use as treatment may pose logistic challenges. CD101 (biafungin, previously SP3025; Cidara Therapeutics, San Diego, CA, USA; Figure 2) is an investigational echinocandin currently under development that has been structurally modified to confer a long half-life (>80 hours), ${ }^{83,84}$ which may allow for less-frequent intravenous administration (e.g., once weekly). This agent has also been shown to be safe without serious adverse effects or withdraws due to adverse effects in healthy volunteers. ${ }^{84}$ In vitro studies have reported potent activity against Candida and Aspergillus species, with a similar low frequency for the development of mutations in hot spot regions of FKS1 and $F K S 2$ as observed with anidulafungin and caspofungin. ${ }^{85,86}$ This in vitro potency has also translated into in vivo efficacy in murine models of invasive candidiasis caused by echinocandin-susceptible and echinocandin-resistant isolates, the later which may be due to the enhanced exposure conferred by the long half-life of this agent. ${ }^{87,88}$ In vitro activity has also been reported for $\mathrm{CD} 101$ against $C$. auris in a small study that included 16 isolates $\left(\mathrm{MIC}_{50} 0.125 \mathrm{mg} / \mathrm{L}, \mathrm{MIC}_{90} 0.25 \mathrm{mg} / \mathrm{L}\right)$, with a potency similar to that of anidulafungin but greater than that of caspofungin and micafungin. ${ }^{89}$

Another glucan synthase inhibitor that is currently being developed for both oral and intravenous administration is SCY-078 (Scynexis, Inc., Jersey City, NJ, USA; Figure 2). Although the mechanism of action of SCY-078 is the same as that of the echinocandins and CD101, this agent is structurally different from the echinocandins and allows for oral administration due to absorption from the gastrointestinal tract. ${ }^{90}$ Similar to the echinocandins, SCY-078 demonstrates potent in vitro activity against various Candida species, including some isolates with known mutations in the FKS1 and FKS2 genes, as well as fluconazole-resistant isolates. ${ }^{90}$ Efficacy has also been demonstrated against infections caused by different Candida species, including C. albicans, C. glabrata, and C. tropicalis, in an established murine model. ${ }^{91}$ Two recent studies have also reported potent in vitro activity of SCY078 against $C$. auris isolates, including inhibition of biofilm formation. ${ }^{92,93}$ Thus, this orally available glucan synthase inhibitor may hold promise against this emerging pathogen. However, as with CD101, in vivo efficacy data against infections caused by $C$. auris are currently lacking. SCY-078 also lacks activity against the Mucorales and Fusarium species, while variable activity has been observed against other molds, including Scedosporium species. ${ }^{94}$

\section{F90 I3 I8 - inhibition of fungal pyrimidine biosynthesis}

The investigational agent F901318 (F2G, Inc., Manchester, UK; Figure 3) inhibits the oxidoreductase enzyme dihydroorotate dehydrogenase, which is important for pyrimidine biosynthesis. ${ }^{95}$ The activity of F901318, a member of the orotomide class of compounds, is fungal specific, as its activity against $A$. fumigatus dihydroorotate dehydrogenase is significantly more potent compared to that of the human enzyme $\left(\mathrm{IC}_{50} 44 \mathrm{nM}\right.$ versus $>100 \mu \mathrm{M}$, respectively). ${ }^{95}$ F901318 has potent in vitro activity against various molds, including Scedosporium species and L. prolificans, as well as endemic fungi (Blastomyces dermatitidis, Coccidioides species, Histoplasma capsulatum).${ }^{96,97}$ Potent in vitro activity has also been observed against Aspergillus species, including azole-resistant $A$. fumigatus that harbors the $\mathrm{TR}_{34} / \mathrm{L} 98 \mathrm{H}$ 

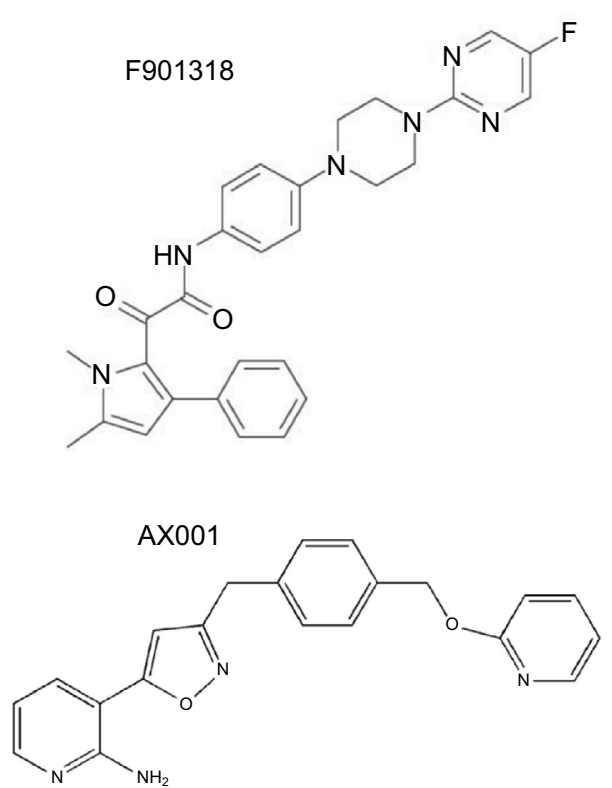

VL-2397

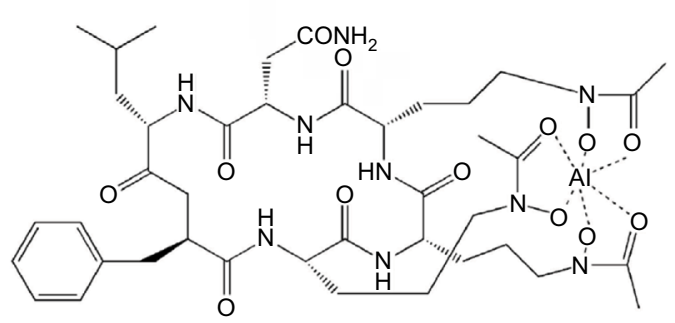

$\mathrm{T}-2307$

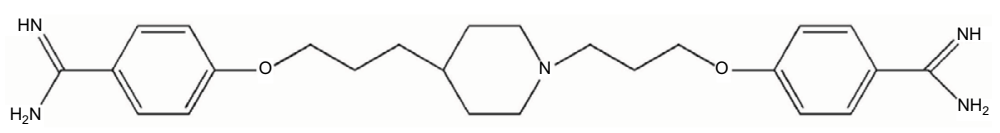

Figure 3 Investigational antifungal agents with novel mechanisms of action, including prevention of fungal pyrimidine via inhibition of dihydroorotate dehydrogenase (F90I3/8), prevention of GPI-anchored protein maturation via inhibition of fungal inositol aceyltransferase (AX00I), collapse of fungal mitochondrial membrane potential (T-2307), and an unknown mechanism of action following transport into fungal cells by the Sit I (VL-2397).

Abbreviations: GPI, glycosylphosphatidylinositol; Sit I, siderophore iron transporter I.

mutation and cryptic Aspergillus species. ${ }^{95,98}$ However, there are significant holes in the spectrum of activity of F901318, as this compound lacks activity against yeast and the members of the order Mucorales. ${ }^{95,96}$ Both oral and intravenous preparations for administration are being developed, and Phase I studies in healthy volunteers have been completed.

\section{AX00I - inhibition of fungal glycosylphosphatidylinositol biosynthesis}

Glycosylphosphatidylinositol (GPI)-anchored proteins serve as adhesions allowing microorganisms to adhere to host mucosal and epithelial surfaces ${ }^{99}$ Thus, GPI-anchored proteins are needed for the establishment of colonization and infection by fungi. AX001 (formerly E1210; Amplyx Pharmaceuticals, San Diego, CA, USA; Figure 3) is an investigational agent that inhibits inositol acyltransferase, thus preventing the maturation of GPI-anchored proteins. ${ }^{100}$ Potent in vitro activity has been reported for AX001 against a broad-spectrum of fungi, including yeast (i.e., Candida species) and molds (i.e., Aspergillus, Fusarium, and Scedosporium species). ${ }^{100-103}$ This in vitro activity has also translated into efficacy in animal models of invasive fungal infections, including invasive candidiasis caused by azole- and echinocandin-resistant isolates, aspergillosis, and fusariosis. ${ }^{104,105}$ Interestingly, AX001 appears to lack in vitro activity against $C$. krusei and the Mucorales, although the in vivo significance of this is not yet known, given its novel mechanism of action in preventing fungal adherence to host surfaces.

\section{T-2307 - collapse of fungal mitochondrial membrane}

T-2307 (Toyama Chemical Co., Toyama, Japan; Figure 3) is an investigational arylamide that is structurally similar to aromatic diamidines. ${ }^{106}$ Exposure to T-2307 causes collapse of fungal mitochondrial membrane potential, and this agent is preferentially taken up by fungal cells compared to mammalian cells by transporter-mediated systems. ${ }^{107,108}$ Thus, T-2307 may offer significant safety advantages over other aromatic diamidines, including pentamidine. Potent in vitro activity has been reported against Candida species, including azole- and echinocandin-resistant isolates of $C$. albicans and C. glabrata, ${ }^{109,110}$ and in vivo efficacy has also been demonstrated in the in vivo models of infections caused by resistant isolates. ${ }^{106,109,110}$ In vitro activity and in vivo efficacy have also been reported against Cryptococcus and Aspergillus species. ${ }^{106}$

\section{VL-2397 - unknown mechanism of action} VL-2397 (formerly ASP2397; Vical Pharmaceuticals, San Diego, CA, USA; Figure 3) is a cyclic metallohexapeptide currently under preclinical development that is structurally related to the siderophore ferrichrome. ${ }^{111}$ Although this agent, which was isolated from an Acremonium species, is able to 
chelate aluminum ions instead of iron, its exact mechanism of action is not fully understood and appears to be independent of aluminum chelation. ${ }^{12}$ However, it is known that it triggers a potent and rapid antifungal effect following transport into fungal cells via siderophore iron transporter 1 , which is absent in mammalian cells. ${ }^{111}$ VL-2397 is active against different fungi, including azole-resistant Aspergillus isolates, which has translated into in vivo efficacy in both silkworm and neutropenic murine models of invasive aspergillosis. ${ }^{113,114}$ A recent study also reported the in vivo efficacy in a neutropenic murine model of invasive candidiasis caused by both wild-type and azole- and echinocandin-resistant $C$. glabrata isolates. ${ }^{115}$ VL-2397 was also well tolerated with sustained levels, but without accumulation in healthy volunteers who received multiple doses per day. ${ }^{116}$

\section{Conclusion}

Clinicians are currently facing several emerging challenges in antifungal resistance. These include increased rates of resistance to azole and echinocandins in several non-C. albicans species and azole resistance in A. fumigatus that may occur due to clinical or environmental exposure to these agents. In addition, there are several species of pathogenic fungi that have reduced susceptibility or frank resistance to many available antifungal agents. Several new antifungals are currently in development that may be more advantageous than the current drugs, both in terms of overcoming antifungal resistance and avoiding adverse effects and drug interactions associated with currently available agents. Continued preclinical evaluation and clinical studies are needed to determine if these agents will be successful in overcoming microbiologic and clinical failure in the setting of antifungal resistance.

\section{Disclosure}

NPW has received grant support from Astellas, bioMerieux, F2G, Merck, Pfizer, and Viamet and has served on the scientific advisory boards for Astellas, Merck, Toyama, and Viamet. The author reports no other conflicts of interest in this work.

\section{References}

1. Vallabhaneni S, Cleveland AA, Farley MM, et al. Epidemiology and risk factors for echinocandin nonsusceptible Candida glabrata bloodstream infections: data from a large multisite population-based candidemia surveillance program, 2008-2014. Open Forum Infect Dis. 2015;2(4):ofv163.

2. Verweij PE, Chowdhary A, Melchers WJ, Meis JF. Azole resistance in Aspergillus fumigatus: can we retain the clinical use of mold-active antifungal azoles? Clin Infect Dis. 2016;62(3):362-368.

3. Rivero-Menendez O, Alastruey-Izquierdo A, Mellado E, CuencaEstrella M. Triazole resistance in Aspergillus spp: a worldwide problem? J Fungi. 2016;2(3):21.
4. Lackner M, Hagen F, Meis JF, et al. Susceptibility and diversity in the therapy-refractory genus scedosporium. Antimicrob Agents Chemother. 2014;58(10):5877-5885.

5. Lackner M, de Hoog GS, Yang L, et al. Proposed nomenclature for Pseudallescheria, Scedosporium and related genera. Fungal Divers. 2014;67(1):1-10.

6. Walsh TJ, Groll A, Hiemenz J, Fleming R, Roilides E, Anaissie E. Infections due to emerging and uncommon medically important fungal pathogens. Clin Microbiol Infect. 2004;10(Suppl 1):48-66.

7. Lockhart SR, Etienne KA, Vallabhaneni S, et al. Simultaneous emergence of multidrug-resistant Candida auris on 3 continents confirmed by whole-genome sequencing and epidemiological analyzes. Clin Infect Dis. 2017;64(2):134-140.

8. Pfaller MA, Diekema DJ, Gibbs DL, et al. Results from the ARTEMIS DISK global antifungal surveillance study, 1997 to 2007: a 10.5-year analysis of susceptibilities of Candida species to fluconazole and voriconazole as determined by CLSI standardized disk diffusion. $J$ Clin Microbiol. 2010;48(4):1366-1377.

9. Pfaller MA, Messer SA, Moet GJ, Jones RN, Castanheira M. Candida bloodstream infections: comparison of species distribution and resistance to echinocandin and azole antifungal agents in intensive care unit (ICU) and non-ICU settings in the SENTRY Antimicrobial Surveillance Program (2008-2009). Int J Antimicrob Agents. 2011;38(1):65-69.

10. Yapar N. Epidemiology and risk factors for invasive candidiasis. Ther Clin Risk Manag. 2014;10:95-105.

11. WHO. Antimicrobial Resistance: Global Report on Surveillance. 2014. Available from: http://www.who.int/drugresistance/documents/ surveillancereport/en/. Accessed June 21, 2017.

12. White TC, Marr KA, Bowden RA. Clinical, cellular, and molecular factors that contribute to antifungal drug resistance. Clin Microbiol Rev. 1998;11(2):382-402.

13. Sanglard D. Emerging threats in antifungal-resistant fungal pathogens. Front Med (Lausanne). 2016;3:11.

14. Oberoi JK, Wattal C, Goel N, Raveendran R, Datta S, Prasad K. Nonalbicans Candida species in blood stream infections in a tertiary care hospital at New Delhi, India. Indian JMed Res. 2012;136(6):997-1003.

15. Beyda ND, John J, Kilic A, Alam MJ, Lasco TM, Garey KW. FKS mutant Candida glabrata: risk factors and outcomes in patients with candidemia. Clin Infect Dis. 2014;59(6):819-825.

16. Alexander BD, Johnson MD, Pfeiffer CD, et al. Increasing echinocandin resistance in Candida glabrata: clinical failure correlates with presence of FKS mutations and elevated minimum inhibitory concentrations. Clin Infect Dis. 2013;56(12):1724-1732.

17. Chakrabarti A, Sood P, Rudramurthy SM, et al. Incidence, characteristics and outcome of ICU-acquired candidemia in India. Intensive Care Med. 2015;41(2):285-295.

18. Adhikary R, Joshi S. Species distribution and anti-fungal susceptibility of Candidaemia at a multi super-specialty center in Southern India. Indian J Med Microbiol. 2011;29(3):309-311.

19. Pfaller MA, Castanheira M, Messer SA, Jones RN. In vitro antifungal susceptibilities of isolates of Candida spp. and Aspergillus spp. from China to nine systemically active antifungal agents: data from the SENTRY antifungal surveillance program, 2010 through 2012. Mycoses. 2015;58(4):209-214.

20. Guo F, Yang Y, Kang Y, et al. Invasive candidiasis in intensive care units in China: a multicentre prospective observational study. J Antimicrob Chemother. 2013;68(7):1660-1668.

21. Xiao M, Fan X, Chen SC, et al. Antifungal susceptibilities of Candida glabrata species complex, Candida krusei, Candida parapsilosis species complex and Candida tropicalis causing invasive candidiasis in China: 3 year national surveillance. J Antimicrob Chemother. 2015;70(3): 802-810.

22. Liao X, Qiu H, Li R, et al. Risk factors for fluconazole-resistant invasive candidiasis in intensive care unit patients: an analysis from the China Survey of Candidiasis study. J Crit Care. 2015;30(4):862. e1-e5. 
23. Pappas PG, Kauffman CA, Andes DR, et al. Clinical practice guideline for the management of candidiasis: 2016 update by the Infectious Diseases Society of America. Clin Infect Dis. 2016;62(4):e1-e50.

24. Messer SA, Diekema DJ, Boyken L, Tendolkar S, Hollis RJ, Pfaller MA. Activities of micafungin against 315 invasive clinical isolates of fluconazole-resistant Candida spp. J Clin Microbiol. 2006;44(2):324-326.

25. Park S, Kelly R, Kahn JN, et al. Specific substitutions in the echinocandin target Fks1p account for reduced susceptibility of rare laboratory and clinical Candida sp. isolates. Antimicrob Agents Chemother 2005;49(8):3264-3273.

26. Garcia-Effron G, Katiyar SK, Park S, Edlind TD, Perlin DS. A naturally occurring proline-to-alanine amino acid change in Fks1p in Candida parapsilosis, Candida orthopsilosis, and Candida metapsilosis accounts for reduced echinocandin susceptibility. Antimicrob Agents Chemother. 2008;52(7):2305-2312.

27. Thompson GR, Wiederhold NP, Vallor AC, Villareal NC, Lewis JS, Patterson TF. Development of caspofungin resistance following prolonged therapy for invasive candidiasis secondary to Candida glabrata infection. Antimicrob Agents Chemother. 2008;52(10):3783-3785.

28. Arendrup MC, Perlin DS. Echinocandin resistance: an emerging clinical problem? Curr Opin Infect Dis. 2014;27(6):484- 492.

29. Malani A, Hmoud J, Chiu L, Carver PL, Bielaczyc A, Kauffman CA. Candida glabrata fungemia: experience in a tertiary care center. Clin Infect Dis. 2005;41(7):975-981.

30. Shields RK, Nguyen MH, Press EG, Updike CL, Clancy CJ. Anidulafungin and micafungin MIC breakpoints are superior to that of caspofungin for identifying FKS mutant Candida glabrata strains and Echinocandin resistance. Antimicrob Agents Chemother. 2013;57(12): 6361-6365

31. Shields RK, Nguyen MH, Press EG, et al. The presence of an FKS mutation rather than MIC is an independent risk factor for failure of echinocandin therapy among patients with invasive candidiasis due to Candida glabrata. Antimicrob Agents Chemother. 2012;56(9):4862-4869

32. Garcia-Effron G, Lee S, Park S, Cleary JD, Perlin DS. Effect of Candida glabrata FKS1 and FKS2 mutations on echinocandin sensitivity and kinetics of 1,3-beta-D-glucan synthase: implication for the existing susceptibility breakpoint. Antimicrob Agents Chemother. 2009;53(9):3690-3699.

33. Arendrup MC, Perlin DS, Jensen RH, Howard SJ, Goodwin J, Hope W. Differential in vivo activities of anidulafungin, caspofungin, and micafungin against Candida glabrata isolates with and without FKS resistance mutations. Antimicrob Agents Chemother. 2012;56(5): 2435-2442.

34. Pfaller MA, Castanheira M, Lockhart SR, Ahlquist AM, Messer SA, Jones RN. Frequency of decreased susceptibility and resistance to echinocandins among fluconazole-resistant bloodstream isolates of Candida glabrata. J Clin Microbiol. 2012;50(4):1199-1203.

35. Healey KR, Zhao Y, Perez WB, et al. Prevalent mutator genotype identified in fungal pathogen Candida glabrata promotes multi-drug resistance. Nat Commun. 2016;7:11128.

36. Chowdhary A, Sharma C, Meis JF. Candida auris: a rapidly emerging cause of hospital-acquired multidrug-resistant fungal infections globally. PLoS Pathog. 2017;13(5):e1006290.

37. Kathuria S, Singh PK, Sharma C, et al. Multidrug-resistant Candida auris misidentified as Candida haemulonii: characterization by Matrix-Assisted Laser Desorption Ionization-Time of Flight Mass Spectrometry and DNA sequencing and its antifungal susceptibility profile variability by Vitek 2, CLSI Broth Microdilution, and Etest Method. J Clin Microbiol. 2015;53(6):1823-1830.

38. Sharma C, Kumar N, Pandey R, Meis JF, Chowdhary A. Whole genome sequencing of emerging multidrug resistant Candida auris isolates in India demonstrates low genetic variation. New Microbes New Infect 2016;13:77-82.

39. Bueid A, Howard SJ, Moore CB, et al. Azole antifungal resistance in Aspergillus fumigatus: 2008 and 2009. J Antimicrob Chemother. 2010;65(10):2116-2118.
40. Howard SJ, Cerar D, Anderson MJ, et al. Frequency and evolution of Azole resistance in Aspergillus fumigatus associated with treatment failure. Emerg Infect Dis. 2009;15(7):1068-1076.

41. Chowdhary A, Sharma C, Kathuria S, Hagen F, Meis JF. Azole-resistant Aspergillus fumigatus with the environmental TR46/Y121F/T289A mutation in India. J Antimicrob Chemother. 2014;69(2):555-557.

42. Chowdhary A, Sharma C, van den Boom M, et al. Multi-azole-resistant Aspergillus fumigatus in the environment in Tanzania. J Antimicrob Chemother. 2014;69(11):2979-2983.

43. Verweij PE, Mellado E, Melchers WJ. Multiple-triazole-resistan aspergillosis. N Engl J Med. 2007;356(14):1481-1483.

44. van der Linden JW, Camps SM, Kampinga GA, et al. Aspergillosis due to voriconazole highly resistant Aspergillus fumigatus and recovery of genetically related resistant isolates from domiciles. Clin Infect Dis. 2013;57(4):513-520.

45. Lockhart SR, Frade JP, Etienne KA, Pfaller MA, Diekema DJ, Balajee SA. Azole resistance in Aspergillus fumigatus isolates from the ARTEMIS global surveillance study is primarily due to the TR/ L98H mutation in the cyp51A gene. Antimicrobl Agents Chemother 2011;55(9):4465-4468.

46. Snelders E, Huis In 't Veld RA, Rijs AJ, Kema GH, Melchers WJ, Verweij PE. Possible environmental origin of resistance of Aspergillus fumigatus to medical triazoles. Appl Environ Microbiol. 2009;75(12):4053-4057.

47. Sugui JA, Peterson SW, Figat A, et al. Genetic relatedness versus biological compatibility between Aspergillus fumigatus and related species. J Clin Microbiol. 2014;52(10):3707-3721.

48. Balajee SA, Gribskov J, Brandt M, Ito J, Fothergill A, Marr KA Mistaken identity: Neosartorya pseudofischeri and its anamorph masquerading as Aspergillus fumigatus. J Clin Microbiol. 2005;43(12): 5996-5999.

49. Ghebremedhin B, Bluemel A, Neumann KH, Koenig B, Koenig W. Peritonitis due to Neosartorya pseudofischeri in an elderly patient undergoing peritoneal dialysis successfully treated with voriconazole. J Med Microbiol. 2009;58(Pt 5):678-682.

50. Varga J, Houbraken J, Van Der Lee HA, Verweij PE, Samson RA. Aspergillus calidoustus sp. nov., causative agent of human infections previously assigned to Aspergillus ustus. Eukaryot Cell. 2008;7(4):630-638.

51. Balajee SA, Kano R, Baddley JW, et al. Molecular identification of Aspergillus species collected for the Transplant-Associated Infection Surveillance Network. J Clin Microbiol. 2009;47(10):3138-3141.

52. Alastruey-Izquierdo A, Mellado E, Pelaez T, et al. Population-based survey of filamentous fungi and antifungal resistance in Spain (FILPOP Study). Antimicrob Agents Chemother. 2013;57(7):3380-3387.

53. Balajee SA, Houbraken J, Verweij PE, et al. Aspergillus species identification in the clinical setting. Stud Mycol. 2007;59:39-46.

54. Cornely OA, Maertens J, Bresnik M, et al. Liposomal amphotericin B as initial therapy for invasive mold infection: a randomized trial comparing a high-loading dose regimen with standard dosing (AmBiLoad trial). Clin Infect Dis. 2007;44(10):1289-1297.

55. Patterson TF, Thompson GR 3rd, Denning DW, et al. Practice guidelines for the diagnosis and management of aspergillosis: 2016 update by the infectious diseases society of America. Clin Infect Dis. 2016;63(4):e1-e60.

56. Johnson LS, Shields RK, Clancy CJ. Epidemiology, clinical manifestations, and outcomes of Scedosporium infections among solid organ transplant recipients. Transpl Infect Dis. 2014;16(4):578-587.

57. Husain S, Munoz P, Forrest G, et al. Infections due to Scedosporium apiospermum and Scedosporium prolificans in transplant recipients: clinical characteristics and impact of antifungal agent therapy on outcome. Clin Infect Dis. 2005;40(1):89-99.

58. Lamaris GA, Chamilos G, Lewis RE, Safdar A, Raad II, Kontoyiannis DP. Scedosporium infection in a tertiary care cancer center: a review of 25 cases from 1989-2006. Clin Infect Dis. 2006;43(12):1580-1584.

59. Nucci M. Emerging moulds: Fusarium, Scedosporium and Zygomycetes in transplant recipients. Curr Opin Infect Dis. 2003;16(6):607-612. 
60. Doligalski CT, Benedict K, Cleveland AA, et al. Epidemiology of invasive mold infections in lung transplant recipients. Am JTransplant. 2014;14(6):1328-1333.

61. Cortez KJ, Roilides E, Quiroz-Telles F, et al. Infections caused by Scedosporium spp. Clin Microbiol Rev. 2008;21(1):157-197.

62. Lewis RE, Wiederhold NP, Klepser ME. In vitro pharmacodynamics of amphotericin B, itraconazole, and voriconazole against Aspergillus, Fusarium, and Scedosporium spp. Antimicrob Agents Chemother. 2005;49(3):945-951.

63. Guarro J, Kantarcioglu AS, Horre R, et al. Scedosporium apiospermum: changing clinical spectrum of a therapy-refractory opportunist. Med Mycol. 2006;44(4):295-327.

64. Kesson AM, Bellemore MC, O'Mara TJ, Ellis DH, Sorrell TC. Scedosporium prolificans osteomyelitis in an immunocompetent child treated with a novel agent, hexadecylphospocholine (miltefosine), in combination with terbinafine and voriconazole: a case report. Clin Infect Dis. 2009;48(9):1257-1261.

65. Biswas C, Sorrell TC, Djordjevic JT, Zuo X, Jolliffe KA, Chen SC. In vitro activity of miltefosine as a single agent and in combination with voriconazole or posaconazole against uncommon filamentous fungal pathogens. J Antimicrob Chemother. 2013;68(12):2842-2846.

66. Nucci M, Marr KA, Queiroz-Telles F, et al. Fusarium infection in hematopoietic stem cell transplant recipients. Clin Infect Dis. 2004;38(9):1237-1242.

67. Nucci M, Marr KA, Vehreschild MJ, et al. Improvement in the outcome of invasive fusariosis in the last decade. Clin Microbiol Infect. 2014;20(6):580-585.

68. O'Donnell K, Sarver BA, Brandt M, et al. Phylogenetic diversity and microsphere array-based genotyping of human pathogenic Fusaria, including isolates from the multistate contact lens-associated U.S. keratitis outbreaks of 2005 and 2006. J Clin Microbiol. 2007;45(7):2235-2248.

69. Hoekstra WJ, Garvey EP, Moore WR, Rafferty SW, Yates CM, Schotzinger RJ. Design and optimization of highly-selective fungal CYP51 inhibitors. Bioorg Med Chem Lett. 2014;24(15):3455-3458

70. Warrilow AG, Hull CM, Parker JE, et al. The clinical candidate VT-1161 is a highly potent inhibitor of Candida albicans CYP51 but fails to bind the human enzyme. Antimicrob Agents Chemother. 2014; 58(12):7121-7127

71. Warrilow AG, Parker JE, Price CL, et al. The Investigational Drug VT-1129 is a highly potent inhibitor of Cryptococcus species CYP51 but only weakly inhibits the human enzyme. Antimicrob Agents Chemother. 2016;60(8):4530-4538.

72. Shubitz LF, Trinh HT, Galgiani JN, et al. Evaluation of VT-1161 for treatment of coccidioidomycosis in murine infection models. Antimicrob Agents Chemother. 2015;59(12):7249-7254.

73. Fothergill AW, Wiederhold NP, Hoekstra WJ, et al. The fungal Cyp51 inhibitors VT-1129 and VT-1161 maintain in vitro activity against Candida albicans isolates with reduced antifungal susceptibility. 51st Interscience Conference on Antimicrobial Agents and Chemotherapy; September 17-20, 2011; Chicago, IL.

74. Long L, Isham N, Ghannoum MA, et al. High in vitro potency of the clinical investigational agent VT-1161 against clinical isolates of Candida spp. 25th European Congress of Clinical Microbiology and Infectious Diseases; April 25-28, 2015; Copenhagen, Denmark.

75. Lockhart SR, Fothergill AW, Iqbal N, et al. The investigational fungal Cyp51 inhibitor VT-1129 demonstrates potent $\mathrm{n}$ vitro activity against Cryptococcus neoformans and Cryptococcus gattii. Antimicrob Agents Chemother. 2016;60(4):2528-2531.

76. Garvey EP, Hoekstra WJ, Schotzinger RJ, Sobel JD, Lilly EA, Fidel PL Jr. Efficacy of the clinical agent VT-1161 against fluconazolesensitive and -resistant Candida albicans in a murine model of vaginal candidiasis. Antimicrob Agents Chemother. 2015;59(9):5567-5573.

77. Najvar LK, Wiederhold NP, Garvey EP, et al. Efficacy of the novel Fungal Cyp51 inhibitor VT-1161 against invasive candidiasis caused by resistant Candida albicans. 18th International Congress of the International Society of Human and Animal Mycoses; June 11-15, 2012; Berlin, Germany.
78. Wiederhold NP, Najvar LK, Garvey EP, et al. Efficacy of the novel fungal Cyp51 inhibitors VT-1161 and VT-1411 against invasive candidiasis caused by Candida albicans and Candida glabrata. 11th American Society of Microbiology Conference on Candida and Candidiasis; March 29 - April 2, 2012; San Francisco, CA.

79. Wiederhold NP, Tran H, Patterson HP, Yates CM, Schotzinger RJ, Garvey EP. The Novel Fungal Cyp51 Inhibitor VT-1598 Demonstrates Potent In vitro Activity against Endemic Fungi, Aspergillus, and Rhizopus. ASM Microbe; June 1-5, 2017; New Orleans, LA.

80. Hargrove TY, Garvey EP, Hoekstra WJ, et al. Crystal structure of the new investigational drug candidate VT-1598 in complex with Aspergillus fumigatus sterol 14alpha-demethylase provides insights into its broad-spectrum antifungal activity. Antimicrob Agents Chemother. 2017;61(7):e00570-17.

81. Wiederhold NP, Tran H, Patterson HP, Yates CM, Schotzinger RJ, Garvey EP. The Novel Fungal Cyp51 Inhibitor VT-1598 Demonstrates Potent In vitro Activity against Candida and Cryptococcus Species. ASM Microbe; June 1-5, 2017; New Orleans, LA.

82. Berkow EL, Le N, Peterson J, et al. In vitro Activity of a Novel CYP51 Inhibitor, VT-1598, against Clinical Isolates of Candida auris. ASM Microbe; June 1-5, 2017; New Orleans, LA.

83. James K, Krishnan R, Smith S, Laudeman C, Polowy K, Vaidya A. Biafungin (CD101), a novel echinocandi, displays a long half-life in the chimpanzee, suggesting a once-weekly IV dosing option (abstr. A-694). 54th Interscience Conference on Antimicrobial Agents and Chemotherapy; September 5-9, 2014; Washington, DC.

84. Sandison T, Ong V, Lee J, Thye D. Safety and pharmacokinetics of CD101 IV, a novel echinocandin, in healthy adults. Antimicrob Agents Chemother. 2017;61(2):e1627-16

85. Pfaller MA, Messer SA, Rhomberg PR, Jones RN, Castanheira M. Activity of a long-acting echinocandin, CD101, determined using CLSI and EUCAST reference methods, against Candida and Aspergillus spp., including echinocandin- and azole-resistant isolates. J Antimicrob Chemother. 2016;71(10):2868-2873.

86. Locke JB, Almaguer AL, Zuill DE, Bartizal K. Characterization of in vitro resistance development to the novel echinocandin CD101 in Candida species. Antimicrob Agents Chemother. 2016;60(10): 6100-6107.

87. Ong V, Hough G, Schlosser M, et al. Preclinical evaluation of the stability, safety and efficacy of CD101, a novel echinocandin. Antimicrob Agents Chemother. 2016;60(11):6872-6879.

88. Zhao Y, Perez WB, Jimenez-Ortigosa C, et al. CD101: a novel longacting echinocandin. Cell Microbiol. 2016;18(9):1308-1316.

89. Ghannoum M, Larkin E, Long L. Susceptibilty of recent Candida auris isolates to the novel echinocandin CD101 and comparator antifungal agents. 27th European Congress on Clinical Microbiology and Infectious Diseases; April 22-25, 2017; Vienna, Austria.

90. Pfaller MA, Messer SA, Motyl MR, Jones RN, Castanheira M. Activity of MK-3118, a new oral glucan synthase inhibitor, tested against Candida spp. by two international methods (CLSI and EUCAST). $J$ Antimicrob Chemother. 2013;68(4):858-863.

91. Lepak AJ, Marchillo K, Andes DR. Pharmacodynamic target evaluation of a novel oral glucan synthase inhibitor, SCY-078 (MK-3118), using an in vivo murine invasive candidiasis model. Antimicrob Agents Chemother. 2015;59(2):1265-1272.

92. Larkin E, Hager C, Chandra J, et al. The emerging pathogen Candida auris: growth phenotype, virulence factors, activity of antifungals, and effect of SCY-078, a novel glucan synthesis inhibitor, on growth morphology and biofilm formation. Antimicrob Agents Chemother. 2017;61(5):e02396-16.

93. Berkow EL, Angulo D, Lockhart SR. In vitro activity of a novel glucan synthase inhibitor, SCY-078, against clinical isolates of Candida auris. Antimicrob Agents Chemother. 2017;61(7):e0435-17.

94. Lamoth F, Alexander BD. Antifungal activities of SCY-078 (MK-3118) and standard antifungal agents against clinical non-Aspergillus mold isolates. Antimicrob Agents Chemother. 2015;59(7):4308-4311. 
95. Oliver JD, Sibley GE, Beckmann N, et al. F901318 represents a novel class of antifungal drug that inhibits dihydroorotate dehydrogenase. Proc Natl Acad Sci U S A. 2016;113(45):12809-12814.

96. Fothergill AW, Wiederhold NP, Sibley G, et al. Spectrum of activity of F901318, the first agent from the newly discovered orotomide class of antifungals (abstr. F-756). Paper presented at: ICAAC/ICC 20152015; San Diego, CA.

97. Wiederhold NP, Law D, Birch M. Dihydroorotate dehydrogenase inhibitor F901318 has potent in vitro activity against Scedosporium species and Lomentospora prolificans. JAntimicrob Chemother. Epub 2017 Mar 15.

98. Buil JB, Rijs AJMM, Meis JFM, et al. Activity of 901318 against azoleresistant and difficult-to-treat Aspergillus species (abstr. P1605). 26th European Congress of Clinical Microbiology and Infectious Diseases; April 9-12, 2016; Amsterdam, the Netherlands.

99. Chaffin WL. Candida albicans cell wall proteins. Microbiol Mol Biol. 2008;72(3):495-544.

100. Miyazaki M, Horii T, Hata K, et al. In vitro activity of E1210, a novel antifungal, against clinically important yeasts and molds. Antimicrob Agents Chemother. 2011;55(10):4652-4658.

101. Pfaller MA, Duncanson F, Messer SA, Moet GJ, Jones RN, Castanheira M. In vitro activity of a novel broad-spectrum antifungal, E1210, tested against Aspergillus spp. determined by CLSI and EUCAST broth microdilution methods. Antimicrob Agents Chemother. 2011;55(11):5155-5158.

102. Pfaller MA, Hata K, Jones RN, Messer SA, Moet GJ, Castanheira M. In vitro activity of a novel broad-spectrum antifungal, E1210, tested against Candida spp. as determined by CLSI broth microdilution method. Diagn Microbiol Infect Dis. 2011;71(2):167-170.

103. Castanheira M, Duncanson FP, Diekema DJ, Guarro J, Jones RN, Pfaller MA. Activities of E1210 and comparator agents tested by CLSI and EUCAST broth microdilution methods against Fusarium and Scedosporium species identified using molecular methods. Antimicrob Agents Chemother. 2012;56(1):352-357.

104. Wiederhold NP, Najvar LK, Fothergill AW, et al. The investigational agent E1210 is effective in treatment of experimental invasive candidiasis caused by resistant Candida albicans. Antimicrob Agents Chemother. 2015;59(1):690-692.

105. Hata K, Horii T, Miyazaki M, et al. Efficacy of oral E1210, a new broad-spectrum antifungal with a novel mechanism of action, in murine models of candidiasis, aspergillosis, and fusariosis. Antimicrob Agents Chemother. 2011;55(10):4543-4551.
106. Mitsuyama J, Nomura N, Hashimoto $\mathrm{K}$, et al. In vitro and in vivo antifungal activities of T-2307, a novel arylamidine. Antimicrob Agents Chemother. 2008;52(4):1318-1324.

107. Shibata T, Takahashi T, Yamada E, et al. T-2307 causes collapse of mitochondrial membrane potential in yeast. Antimicrob Agents Chemother. 2012;56(11):5892-5897.

108. Nishikawa H, Yamada E, Shibata T, et al. Uptake of T-2307, a novel arylamidine, in Candida albicans. J Antimicrob Chemother. 2010;65(8):1681-1687.

109. Wiederhold NP, Najvar LK, Fothergill AW, et al. The novel arylamidine T-2307 maintains in vitro and in vivo activity against echinocandin-resistant Candida albicans. Antimicrob Agents Chemother 2015;59(2):1341-1343.

110. Wiederhold NP, Najvar LK, Fothergill AW, et al. The novel arylamidine T-2307 demonstrates in vitro and in vivo activity against echinocandin-resistant Candida glabrata. J Antimicrob Chemother. 2016;71(3):692-695.

111. Nakamura I, Ohsumi K, Yoshimatsu K, et al. ASP2397: a novel natural prodcut with potent fungicidal activity against Aspergillus spp. - a new mode of action and in vitro activity (abstr. F-1590). 54th Interscience Conference on Antimicrobial Agents and Chemotherapy; September 5-9, 2014; Washington, DC.

112. Nakamura I, Yoshimura S, Masaki T, et al. ASP2397: a novel antifungal agent produced by Acremonium persicinum MF-347833. J Antibiot (Tokyo). 2016;7:1-7.

113. Nakamura I, Kanasaki R, Yoshikawa K, et al. Discovery of a new antifungal agent ASP2397 using a silkworm model of Aspergillus fumigatus infection. J Antibiot (Tokyo). 2016;70(1):41-44.

114. Nakamura I, Nakai T, Matsumoto S, et al. ASP2397: a novel natural product with potent fungicidal activity against Aspergillus spp. - In vivo activity against A. fumigatus. 54th Interscience Conference on Antimicrobial Agents and Chemotherapy; September 5-9, 2014; Washington, DC

115. Wiederhold NP, Najvar LK, Jaramillo R, et al. The Novel Antifungal VL-2397 Demonstrates Efficacy in an In vivo Model of Invasive Candidiasis caused by Wild-Type and Multi-Drug Resistant Candida glabrata. ASM Microbe; June 1-5, 2017; New Orleans, LA.

116. Mammen MP, Armas DR, Smith LR, et al. Phase 1 Safety and Pharmacokinetic Study of VL-2397, a Novel Antifungal Agent. ASM Microbe; June 1-5, 2017; New Orleans, LA.
Infection and Drug Resistance

\section{Publish your work in this journal}

Infection and Drug Resistance is an international, peer-reviewed openaccess journal that focuses on the optimal treatment of infection (bacterial, fungal and viral) and the development and institution of preventive strategies to minimize the development and spread of resistance. The journal is specifically concerned with the epidemiology of antibiotic

\section{Dovepress}

resistance and the mechanisms of resistance development and diffusion in both hospitals and the community. The manuscript management system is completely online and includes a very quick and fair peerreview system, which is all easy to use. Visit http://www.dovepress.com/ testimonials.php to read real quotes from published authors. 